\title{
EVALUACIÓN DE ESTEROIDES SEXUALES FECALES DEL PAVÓN CORNUDO (OREOPHASIS DERBIANUS, AVES: CRACIDAE) EN CAUTIVERIO
}

\author{
LuIs M. GARCÍA-FERIA* \& CAROLINA VALDESPINO
}

Red de Biología y Conservación de Vertebrados. Instituto de Ecología, A. C. Carretera antigua a Coatepec No. 351, El Haya. C.P. 91070, Xalapa, Veracruz, México. < luizoo@yahoo.com>

Recibido: 20/02/2013; aceptado:22/09/2014

\begin{abstract}
García-Feria, L. M. \& Valdespino, C. 2015. Evaluación de esteroides sexuales fecales del pavón cornudo (Oreophasis derbianus, Aves: Cracidae) en cautiverio. Acta Zoológica Mexicana (n. s.), 31(1): $1-9$.
\end{abstract}

RESUMEN. La cuantificación de esteroides sexuales a lo largo del tiempo permite evaluar cambios en la función reproductiva de los individuos. En este trabajo se describen los perfiles de testosterona fecal $\left(T_{f}\right)$ y estradiol fecal $\left(E_{2 f}\right)$ de tres machos y $E_{2 f}$ de cuatro hembras de Oreophasis derbianus (Aves: Cracidae) dentro de un programa de reproducción. Los esteroides sexuales se cuantificaron de muestras fecales mediante un inmunoensayo por quimioluminiscencia. No se encontraron diferencias en las concentraciones de $T_{f}$ entre los machos $(P=0.894)$ ni a lo largo del tiempo de muestreo $(P=0.305)$. Los perfiles hormonales a través del tiempo sugieren un sistema de apareamiento monógamo, aún cuando se ha reportado la poliginia en esta ave. Uno de los machos presentó mayores concentraciones de $\mathrm{E}_{2 \mathrm{f}}$ $(34.09 \pm 1.08 \mathrm{ng} / \mathrm{g})$ que los otros dos $(6.83 \pm 1.38$ y $7.98 \pm 2.13 \mathrm{ng} / \mathrm{g})$, lo que sugiere una edad reproductiva mayor, ya que previamente se ha reportado que esta hormona se incrementa en la senescencia. Las hembras tampoco mostraron diferencias significativas entre ellas $(P=$ $0.473)$ o a través del tiempo de muestreo $(P=0.668)$. La evaluación de las concentraciones de ambos esteroides en el mismo individuo y su cociente permitió definir que la actividad hormonal corresponde a la tradicionalmente asignada a cada sexo con los machos mostrando las concentraciones de $\mathrm{T}_{\mathrm{f}}$ mayores que las hembras y lo contrario en el caso del $E_{2 f}$. La relación $E_{2 f} / T_{f}$ es por lo tanto mayor en las hembras. En cautiverio, estimar la actividad gonadal para determinar el éxito o la falla reproductiva, evaluar la madurez sexual y la fertilidad de los animales silvestres, permite determinar si un individuo o un grupo cuentan con la capacidad de reproducirse y formar parte de un programa de reproducción.

Palabras clave: Oreophasis derbianus, esteroides fecales, muestreo no invasivo, monogamia, senescencia.

\section{INTRODUCCIÓN}

El pavón cornudo, Oreophasis derbianus Gray 1844, es una de las ocho especies de crácidos que habitan en México, catalogado en peligro de extinción y de alta prioridad de conservación (Brooks \& Strahl 2000). La población estimada es de entre 1000 y 2500 individuos, y la cautiva de 72 individuos reportados hasta el 2008 (Cornejo 2008, 2009). Los programas de reproducción son una estrategia para la recuperación de las especies cuyas poblaciones silvestres se encuentran en estado crí-
García-Feria, L. M. \& Valdespino, C. 2015. Evaluation of fecal sex steroids in captive horned guan (Oreophasis derbianus, Aves: Cracidae). Acta Zoológica Mexicana (n. s.), 31(1): 1-9.

ABSTRACT. The measurement of sexual steroids over time allows evaluating the changes in reproductive function of individuals. We evaluate the profiles of fecal testosterone $\left(T_{f}\right)$ and fecal estradiol $\left(E_{2 f}\right)$ of three males and four females of Oreophasis derbianus (Aves: Cracidae) in a breeding program. Sex steroids faecal samples were quantified by a chemoluminiscence immunoassay. There are not significant differences in the $\mathrm{T}_{\mathrm{f}}$ concentrations between males $(P=0.894)$ and along the sampling time ( $P=0.305)$, but there was a hormone pattern corresponding with monogamous mating system, even when the polygyny has been reported in this bird. One of the individuals had higher concentrations $E_{2 f}(34.09 \pm 1.08 \mathrm{ng} / \mathrm{g}$ ) than the other two (6.83 \pm 1.38 and $7.98 \pm 2.13 \mathrm{ng} / \mathrm{g}$ ), suggesting a greater reproductive age, as has been previously reported that this hormone increases with senescence. The females did no show significant differences between them $(P=0.473)$ and along sampling time $(P=0.668)$. The evaluation of the concentrations of both steroids in the same individual and their ratio allowed defining that the hormonal activity corresponds to the traditionally assigned to each sex, where males show $\mathrm{T}_{\mathrm{f}}$ concentrations larger than females and the opposite in the case of $E_{2 f}$. The $E_{2 f} / T_{f}$ ratio is therefore greater in females. In captivity, estimate the gonadal activity to determine the success or reproductive failure, assess sexual maturity and fertility of wild animals, allow determining if an individual or group has the ability to reproduce and become part of a breeding program.

Key words: Oreophasis derbianus, fecal steroids, non invasive sampling, monogamy, senescence.

tico; su objetivo principal es el logro del máximo éxito reproductivo (Sheppard 1995, Estudillo 1997). En México, zoológicos y criaderos han manteniendo parejas potencialmente reproductoras desde 1994, pero algunas no se han reproducido, probablemente por el contenido de grasa en la dieta como factor crítico (Cornejo 2009). Aunque se desconocen las causas reales de la falla reproductiva, la estrategia de formación de parejas reproductivas ha sido funcional a partir del 2002 con el programa de reproducción intensivo; no obstante, se han reproducido solo el 58\% de los individuos y el 32\% de los po- 
llos obtenidos provienen de uno de ellos (Cornejo 2009).

En los programas de reproducción en cautiverio, uno de los primeros obstáculos es la determinación del sexo cuando no existe un dimorfismo. Existen algunos crácidos con dimorfismo sexual muy evidente, donde los machos presentan una tráquea muy alongada (Delacour \& Amadon 1973). En otro caso, Oreophasis derbianus es una especie monomórfica, ya que no presenta caracteres intersexuales bien diferenciados (Vaurie 1968), pero se menciona que las hembras tienen el cuerno ligeramente más corto (Álvarez del Toro 1976). Algunos autores indican que el tamaño del cuerno, más que demostrar dimorfismo sexual, probablemente se asocie con la edad del individuo o corresponda a la variación genética individual (González-García 1984).

La determinación del sexo se ha realizado mediante la identificación de cromosomas sexuales y la laparotomía para observar directamente las gónadas (Ritchie et al. 1994); técnicas moleculares para la detección de los genes CHD (Griffiths et al. 1998), que en O. derbianus no ha sido exitosa con estos marcadores (Bermúdez-Humarán et al. 2002). También se ha determinado el sexo en Oreophasis mediante la diferenciación de las vocalizaciones emitidas por individuos de cada sexo (González-García 1984), y últimamente se ha realizado por la eversión del aparato reproductor de los polluelos de hasta un mes de edad, pero no ha resultado confiable para la identificación de las hembras (Cornejo 2009). Así que la determinación de la proporción de hormonas sexuales estrógenos: andrógenos es una buena opción para la determinación de sexos, exclusivamente en aves adultas (Bercovitz 1987; Estudillo 1997; Ritchie et al. 1994), que ya están en edad reproductiva; asimismo, con esta determinación se puede evaluar su actividad gonadal, debido a que la interacción de las hormonas reproductivas $\left(\mathrm{E}_{2} / \mathrm{T}\right)$ controla el sistema reproductor e influye en el éxito reproductivo del individuo (Gee 1995).

El conocimiento de los patrones endocrinos permite determinar la condición y capacidad reproductiva al evaluar la actividad gonadal (Schwarzenberger \& Brown 2013). Por lo tanto, es de suma importancia estimar la actividad gonadal para determinar el éxito o la falla reproductiva, así como para evaluar la madurez sexual y la fertilidad de los individuos. Al conocer estas características en una especie se puede determinar si un individuo o un grupo cuentan con la capacidad de reproducirse (Lasley \& Kirkpatrick 1991, Gee 1995, Sheppard 1995), ya sea por reproducción natural o por técnicas de reproducción asistida (Cockrem \& Rounce 1995, Staley et al. 2007).

Todos los individuos producen esteroides sexuales (estrógenos y andrógenos) pero en diferentes concentra- ciones; los machos producen y secretan más andrógenos mientras que las hembras más estrógenos. La relación de los estrógenos (por ejemplo el estradiol, $\mathrm{E}_{2}$ ) respecto a los andrógenos (por ejemplo la testosterona, T) refleja cambios en la función reproductiva a lo largo del tiempo (Johnson 1999, Kirby \& Froman 1999, Nelson 2000). En las hembras, los estrógenos se crean en las células intersticiales de la granulosa del ovario, convirtiendo la $\mathrm{T}$ y la androstenediona en $E_{2}$ (Nelson 2000), mientras que en los machos, el $\mathrm{E}_{2}$ es aromatizado en el cerebro a partir de la $\mathrm{T}$, pero también se origina en los testículos y tiene un papel importante en reducción de la fertilidad. Por ejemplo, en los pollos domésticos machos el $\mathrm{E}_{2}$ plasmático se incrementa mientras que la $\mathrm{T}$ decrece conforme avanza la edad, por lo que estos cambios se asocian con el decremento de la fertilidad (Weil et al. 1999). Conociendo este patrón de interacciones hormonales, nos planteamos: 1. Evaluar la función reproductiva del pavón cornudo para detectar los eventos reproductivos; 2. Registrar cambios de comportamiento asociados a la reproducción (vocalizaciones, montas y puesta de huevos); y 3. Medir las concentraciones de testosterona y estradiol fecales de tres machos y estradiol fecal de cuatro hembras de Oreophasis derbianus que formaban parte de un programa de reproducción en cautiverio.

\section{MATERIALES Y MÉTODOS}

Se estudiaron tres machos (M1, M2 y M3) y cuatro hembras (H1, H2, H3 y H4) adultos de O. derbianus. Los tres machos y tres de las hembras (H1-3) fueron capturados en 1994 para su reproducción en cautiverio. La cuarta hembra (H4) nació en cautiverio en 1999 (Cornejo 2008). Estos pavones cornudos se encontraban en el área de reproducción de aves del Zoológico Africam Safari, Puebla, México (exentos de la exhibición al público), conformando parejas (H1/M1, H2/M2) y un trío reproductor (H3 y H4/M3), mantenidos en albergues de malla ciclónica de 6 x 8 x 2.5 metros. Los machos M1 y M2 se han reproducido exitosamente desde 1998, pero no existían reportes de que el macho perteneciente al trío (M3) se hubiera reproducido en cautiverio. Los albergues estaban divididos en una zona interior ( $6 \times 2$ metros) con piso de cemento y techo de lámina de asbesto, y una zona exterior de piso de tierra y hojarasca con plantas y enredaderas para disminuir la visibilidad hacia albergues adyacentes, la exposición directa al sol, las corrientes de aire y a la actividad de los trabajadores.

Entre septiembre del 2001 y julio del 2002 se colectaron mensualmente entre una y seis muestras fecales de cada individuo y se registraron conductas reproductivas como vocalizaciones de cortejo, montas y la puesta de los 
huevos por las hembras, permitiendo delimitar la temporada reproductiva. La colecta de las excretas se realizó entre las 7:00 y las 12:00 horas, con intervalos de $30 \mathrm{mi}-$ nutos entre albergues o hasta la obtención de la muestra. Las muestras fueron almacenadas en bolsas plásticas, refrigeradas por dos horas y luego congeladas a $-20^{\circ} \mathrm{C}$ hasta su procesamiento en el laboratorio. La extracción hormonal, de acuerdo al método previamente reportado por Bishop \& Hall (1991) y Cockrem \& Rounce (1994, 1995), se llevó a cabo en el Laboratorio de Fisiología de la Reproducción del Centro de Investigaciones Fisiológicas de la Universidad Autónoma de Tlaxcala. Las concentraciones de estradiol fecal $\left(E_{2 f}\right)$ y testosterona fecal $\left(T_{f}\right)$ fueron cuantificadas mediante inmunoensayos por quimioluminiscencia (Weeks \& Woodhead 1984, Kricka 1984, 1991) con los kits IMMULITE Estradiol e IMMULITE Total Testosterone (Diagnostic Products Corporation, Los Angeles, CA, USA) en el Laboratorio de Neurofisiología de la Universidad Autónoma de Querétaro. Los anticuerpos de diagnóstico tienen 100\% de especificidad y baja reacción cruzada con otros esteroi-

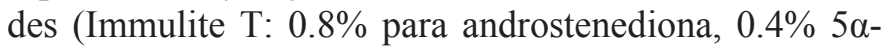
androstan-3 $\beta, 17 \beta$-diol, 2.4\% $5 \alpha$-dihidro-testosterona y $0.8 \%$ para metiltestosterona; Immulite E: $0.032 \%$ con $17 \alpha$ estradiol, 0.535 con estriol, $2.09 \%$ con estrona, $1.22 \%$ con etinil estradiol y 17 esteroides más con menos del $0.1 \%$ ). Los mayores valores de los coeficientes de variación intraensayo e interensayo detectados por el fabricante son del $9.5 \%$ y del $16 \%$, respectivamente. Esto demuestra una imprecisión en la cuantificación hormonal del 4.5\% y 6\%, respectivamente (recomendado: CV intraensayo $<5 \%$, CV interensayo <10\%) (Casanueva \& Vázquez 1995).

Las concentraciones fecales de los metabolitos de $E_{2 f}$ y $T_{f}$ están expresadas en nanogramos por gramo de excreta en peso seco (ng/g) y se expresan con el valor de la media y el error estándar. Las fechas en todos los casos siguen el formato día/mes/año.
Los perfiles hormonales de cada individuo fueron comparados en el periodo de colecta con un análisis de varianza de medidas repetidas (ANOVA-MR; von Ende 2001) con diseño de un factor (tiempo) y 11 niveles (meses) siendo la variable dependiente la concentración hormonal. De igual manera, los valores totales de $E_{2 f}$ de los machos fueron comparados con una ANOVA-MR utilizando una comparación múltiple post-hoc con el método de Holm-Sidak para definir las diferencias particulares. También se realizó una prueba de Chi Cuadrada para conocer si los valores promedio de las relaciones de $\mathrm{E}_{2 \mathrm{f}} / \mathrm{T}_{\mathrm{f}}$ de cada individuo (como evaluación de la función reproductiva) eran valores esperados para crácidos monomórficos ( $<0.3$ para machos y $>0.6$ para hembras; según Czekala \& Lasley 1977) y una prueba de t para muestras independientes para comparar entre sexos. Además, se realizó un análisis de conjuntos difusos (Bosserman \& Ragade 1982) para reconocer la correspondencia sexual de la $E_{2 f} / T_{f}$. Los análisis estadísticos se realizaron con el programa STATISTICA versión 7.0 (StatSoft, Inc. 2004) y se utilizó un nivel de significancia de 0.05. El análisis de conjuntos difusos se realizó con el programa de Fuzzy Clustering Tool versión 2 (Equihua-Zamora 2001).

\section{RESULTADOS}

El registro de comportamientos tales como vocalizaciones, cópulas y puestas, permitieron definir la temporada reproductiva, la cual inició en febrero, con la primera vocalización de cortejo de los machos ("mooing”; 13/03/2002) y concluyó en abril con el último mugido emitido (18/04/2002). No se incluyó la fase de incubación, ya que los huevos fueron retirados para ser incubados artificialmente. El resto del año se consideró como periodo no reproductivo.

Perfil hormonal de machos. El número de muestras analizadas varió por individuo (Cuadro 1). Los perfiles de $T_{f}$ de los machos muestran un pico al inicio de la temporada

Cuadro 1. Número de muestras fecales, concentraciones medias de $\mathrm{T}_{\mathrm{f}} \mathrm{y} \mathrm{E}_{2 \mathrm{f}} \mathrm{y}$ probabilidad de asignación al sexo de tres machos y cuatro hembras de Oreophasis derbianus cautivos.

\begin{tabular}{|c|c|c|c|c|c|c|c|c|c|}
\hline Individuo & $\mathrm{n}$ & $\mathrm{T}_{\mathrm{f}}$ & $\pm \mathrm{EE}$ & $\mathrm{n}$ & $E_{2 f}$ & $\pm \mathrm{EE}$ & $\mathrm{E}_{2 \mathrm{f}} / \mathrm{T}_{\mathrm{f}}$ & $\begin{array}{c}\text { p Conjunto } \\
1^{*}\end{array}$ & $\begin{array}{c}\text { p Conjunto } \\
2^{*}\end{array}$ \\
\hline M1 & 16 & 116.21 & 29.02 & 7 & 7.69 & 1.09 & 0.066 & 0.972 & 0.028 \\
\hline M3 & 8 & 92.97 & 19.19 & 3 & 34.09 & 1.09 & 0.367 & 0.833 & 0.167 \\
\hline $\mathrm{H} 1$ & 15 & 20.64 & 4.09 & 18 & 13.18 & 2.93 & 0.639 & 0.071 & 0.929 \\
\hline $\mathrm{H} 4$ & 4 & 67.65 & 20.50 & 9 & 16.45 & 3.69 & 0.243 & 0.272 & 0.728 \\
\hline
\end{tabular}

* Probabilidad de asignación al sexo por medio de análisis de conjuntos difusos a partir de las concentraciones medias de $\mathrm{T}_{\mathrm{f}}, \mathrm{E}_{2 \mathrm{f}} \mathrm{y}$ la relación $\mathrm{E}_{2 \mathrm{f}} / \mathrm{T}_{\mathrm{f}}$. 



Figura 1. Perfil de $\mathrm{T}_{\mathrm{f}} \mathrm{y} \mathrm{E}_{2 \mathrm{f}}$ de tres pavones (Oreophasis derbianus) machos adultos. La gráfica de M1 y M2 corresponde a los machos pertenecientes a parejas; la gráfica de M3 corresponde al macho del trío. El valor de cada mes es la media \pm error estándar. Los triángulos blancos indican la primera vocalización de cortejo y los triángulos negros la última vocalización emitida. El área sombreada indica el periodo reproductivo registrado.

no reproductiva (Fig. 1), en octubre para el macho M1 (144.3 $\pm 76.1 \mathrm{ng} / \mathrm{g})$ y en noviembre para los machos M2 (156 \pm 127.2 ng/g) y M3 (181.6 ng/g). La temporada reproductiva inició con las vocalizaciones de cortejo entre febrero y marzo, correspondiendo con incrementos de la $\mathrm{T}_{\mathrm{f}}$ (M1, $235.73 \pm 84.26 \mathrm{ng} / \mathrm{g} ; \mathrm{M} 2,136.72 \pm 40.45 \mathrm{ng} / \mathrm{g}$ ). Las montas ocurrieron, para M1, el 28/03/2001y para M2, el 09/03/2001. Las concentraciones de $\mathrm{T}_{\mathrm{f}}$ disminuyeron considerablemente (M1, $131.28 \pm 65.77$ ng/g; M2, 21.52 $\pm 5.2 \mathrm{ng} / \mathrm{g}$ ) para cuando las hembras produjeron los huevos (30/03/2001 y 02/04/2001 para la hembra del M1, y 22 y 25/03/2011 para la hembra del M2). El M3 presentó dos incrementos en la $\mathrm{T}_{\mathrm{f}}$, el primero en temporada no reproductiva (181.6 ng/g) y el segundo en la temporada reproductiva (156.4 ng/g) y no efectuó ninguna vocalización de cortejo, ni mostró interés por las hembras. No hubo diferencias significativas entre los promedios mensuales de $\mathrm{T}_{\mathrm{f}}$ de los machos $\left(F_{2,27}=0.113 ; \mathrm{p}=0.894\right)$, ni durante los 11 meses de muestreo (ANOVA-MR, $F_{10,27}$ $=1.318 ; \mathrm{p}=0.305)$. En contraste, la concentración de $\mathrm{E}_{2 \mathrm{f}}$ fue significativamente mayor en M3 (34.48 $\pm 1.64 \mathrm{ng} / \mathrm{g})$ $\left(F_{2,7}=24.61 ; \mathrm{p}=0.039\right.$; Holm-Sidak Test, M1/M2: $\mathrm{p}=$ 0.204, M1/M3: $\mathrm{p}=0.024$, M2/M3: $\mathrm{p}=0.042$; Fig. 2).

Perfil hormonal de hembras. Los perfiles de $E_{2 f}$ de las cuatro hembras (Fig. 3) no tuvieron diferencias significativas entre individuos $(\mathrm{H}=2.510$, g.l. $=3, \mathrm{p}=0.473)$, ni a lo largo del tiempo (ANOVA-MR, $F_{10,37}=0.756$, $\mathrm{p}=$ 0.668). La hembra $\mathrm{H} 1$ presentó un incremento en diciembre (35.19 $\pm 18 \mathrm{ng} / \mathrm{g})$, disminuyendo subsecuentemente. En la temporada reproductiva, las concentraciones fueron bajas durante el cortejo, la cópula (28/03/2002) y la puesta de dos huevos (30/03/2002 y 02/04/2002). Para la hembra $\mathrm{H} 2$, el $\mathrm{E}_{2 \mathrm{f}}$ mostró un incremento en noviembre (48.21 $\pm 44.95 \mathrm{ng} / \mathrm{g})$ disminuyendo gradualmente y manteniéndose bajo durante la temporada reproductiva (entre 5.26 y 25.12 ng/g). Durante el periodo de vocalización de cortejo del macho (13/02/2002 a 18/04/2002), las concentraciones de $\mathrm{E}_{2 \mathrm{f}}$ fueron bajas comparadas con los observadas en la temporada no reproductiva.

La hembra $\mathrm{H} 3$ no mostró cambios notables en la $\mathrm{E}_{2 \mathrm{f}}$, aunque en septiembre presentó un pico (53.33 ng/g) (Fig.



Figura 2. Comparación de los niveles de $\mathrm{E}_{2 \mathrm{f}}$ en tres pavones, Oreophasis derbianus, machos adultos. M1 = macho de la pareja 1; M2 = macho de la pareja 2; y M3 = macho del trío. 


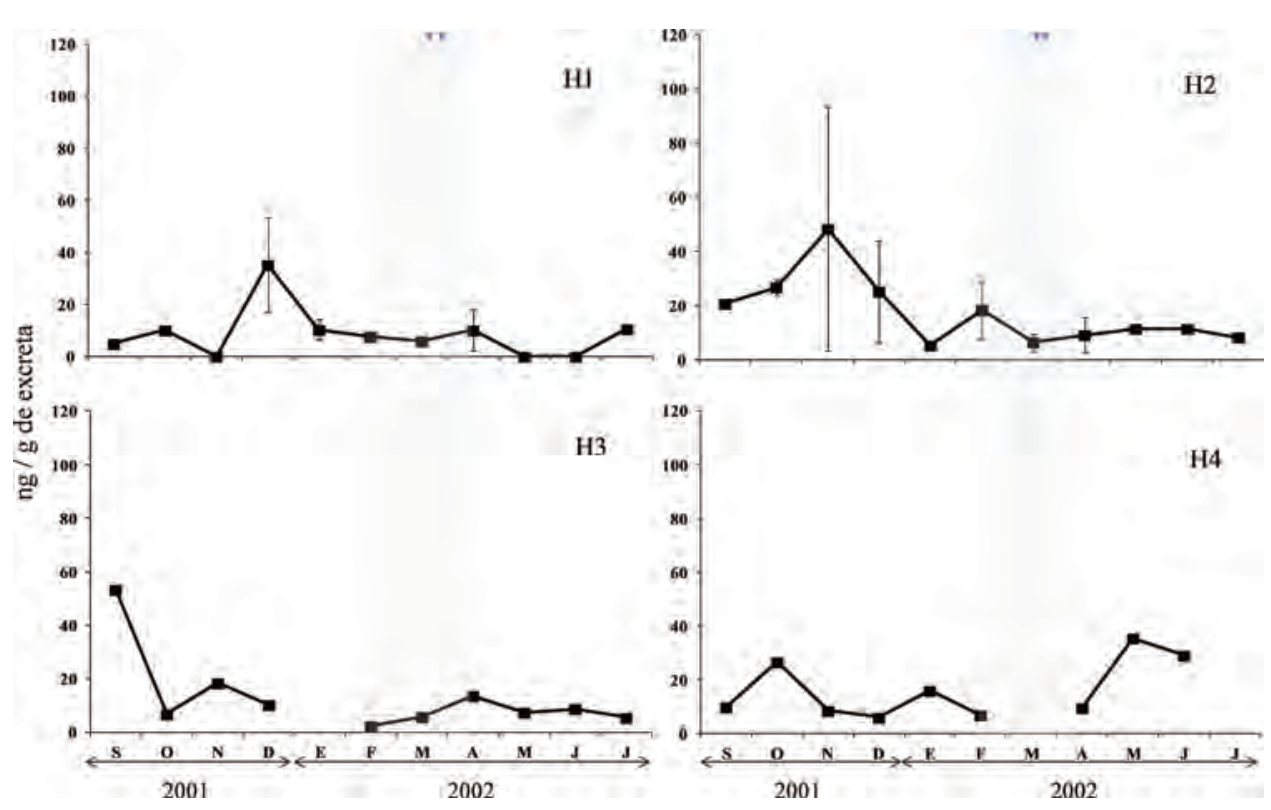

Figura 3. Perfil de estradiol fecal $\left(\mathrm{E}_{2 \mathrm{f}}\right)$ de cuatro pavones (Oreophasis derbianus) hembras adultas. La gráfica de $\mathrm{H} 1$ y $\mathrm{H} 2$ corresponde a las hembras pertenecientes a parejas; la gráfica de H3 y H4 corresponden a las hembras del trío. El valor de cada mes es la media \pm error estándar. El área sombreada indica la temporada reproductiva observada. Para H1 y H2, los triángulos blancos indican la fecha de la cópula y los triángulos negros indican las fechas de las puestas.

3). Esta hembra no exhibió comportamiento reproductivo alguno, aún cuando la edad calculada a la fecha del muestreo era de diez años. Se ha reportado que la edad reproductiva para las hembras es de 14 años (Cornejo 2009), y la edad máxima reportada de sobrevivencia en cautiverio para la especie fue de 15 años (Álvarez del Toro 1976, Cornejo 2008). Por otro lado, la H4 mostró un pequeño aumento de $E_{2 f}$ en octubre (26.62 ng/g), mantuvo concentraciones bajas durante la temporada reproductiva que aumentaron para mayo (35.49 ng/g) y disminuyeron posteriormente. Esta hembra presentó comportamientos agresivos hacia el macho (M3) en diciembre del 2001. A inicios de febrero del 2002, comenzó a hurgar el material del nido, acomodándolo al tamaño y forma de su cuerpo echado. Además amenazaba al M3 y a la H3 cuando se acercaban al nido; no obstante, no realizó ninguna puesta.

Relación estradiol/testosterona fecales. Los coeficientes de relación $\mathrm{E}_{2 \mathrm{f}} / \mathrm{T}_{\mathrm{f}}$ no fueron significativamente diferentes respecto de los valores esperados de $<0.3$ para machos y $>0.6$ para hembras de crácidos monomórficos según Czekala \& Lasley (1977) (Chi-cuadrada $=0.86$, g.l. $=6$, p $=0.99)$, ni entre sexos $(\mathrm{t}=-1.58$, g.l. $=5, \mathrm{p}=0.17)$. Por otro lado, el análisis de conjuntos difusos mostró correspondencia de la relación hormonal y las concentraciones medias de $\mathrm{T}_{\mathrm{f}}$ y $\mathrm{E}_{2 \mathrm{f}}$ con el sexo del individuo (Cuadro 1, Fig. 4). Los centroides de los conjuntos difusos para los machos fueron de 106.05 para $T_{f}$, de 14.62 para $E_{2 f} y$ de
0.15 para la relación $\mathrm{E}_{2 \mathrm{f}} / \mathrm{T}_{\mathrm{f}}$, mientras que para las hembras fueron de 44.16 para $\mathrm{T}_{\mathrm{f}}$, para $\mathrm{E}_{2 \mathrm{f}}$ de 15.04 y la relación $\mathrm{E}_{2 \mathrm{f}} / \mathrm{T}_{\mathrm{f}}$ de 0.39 .

\section{DISCUSIÓN}

En resumen, la temporada reproductiva del pavón en cautividad inició en febrero con las primeras vocalizaciones



Figura 4. Segregación en conjuntos difusos y correspondencia al sexo de las relaciones $E_{2 f} / T_{f} y$ las concentraciones medias de $T_{f} y$ $\mathrm{E}_{2 \mathrm{f}}$ de tres machos y cuatro hembras de Oreophasis derbianus. Los triángulos corresponden a las hembras y los rombos a los machos. 
de cortejo de los machos y terminó a mediados de abril, similar a lo reportado en vida libre (González-García 1984, 1995). Esto podría sugerir que la latitud no influyó en el comportamiento reproductivo, ya que no existió una asincronía en los comportamientos (Moore et al. 2005, Garamszegi et al. 2008). Probablemente, los incrementos de la $\mathrm{T}_{\mathrm{f}}$ correspondan a las primeras vocalizaciones $\mathrm{y}$ disminuyen después de las cópulas y de la puesta de los huevos por las hembras. Las hembras tuvieron concentraciones de $\mathrm{E}_{2 \mathrm{f}}$ similares, no obstante las de las hembras $\mathrm{H} 1 \mathrm{y} \mathrm{H} 2$ fueron altas previo a la temporada reproductiva. El coeficiente de la relación $\mathrm{E}_{2 \mathrm{f}} / \mathrm{T}_{\mathrm{f}}$ permite la asignación al sexo y da un conocimiento de la actividad gonadal, ya que un cociente mayor sugeriría una hembra y uno menor a un macho, y cualquier desbalance pudiera sospecharse de una anormalidad. Además, tuvimos el hallazgo de que el macho M3 posiblemente sea de mayor edad a lo considerado cuando fue capturado en vida libre, ya que las concentraciones de $\mathrm{E}_{2}$ se incrementan con la edad.

Perfil hormonal de machos. En los pavones, el incremento de la $\mathrm{T}_{\mathrm{f}}$ en la temporada reproductiva coincidió con la aparición de las vocalizaciones de cortejo (febrero) como lo reportado en vida libre (González-García 1984, 1995). Su disminución ocurre después de las cópulas y puesta de los huevos por las hembras, lo que muestra una tendencia similar a los patrones generales de monogamia descritos en aves (Wingfield 1984, Wingfield et al. 1990). Podría sugerirse que los picos de $T_{f}$ previos a la temporada reproductiva son reflejo del desarrollo y activación gonadal (Blas et al. 2010, Williams 2012, Gaur et al. 2013). Aunque son pocos los individuos estudiados, la tendencia al patrón monógamo observado no concuerda con el de individuos silvestres, en los que un macho puede copular con 3 o 4 hembras diferentes, ajustándose al sistema polígamo serial (González-García 1984, 1995). Las posibles explicaciones de este perfil monógamo son: 1) las prácticas de manejo en cautiverio (tamaño del albergue y/o dieta), dado que en otras especies se ha visto este cambio de sistema de apareamiento asociado al cautiverio (Donham 1980, Haase \& Donham 1980); 2) posible “umbral poligínico” (Ptak \& Lachmann 2003), la escasez de recursos (alimento, espacio y/o hembras) puede promover asociaciones monógamas (Emlen \& Oring 1977, Leslier et al. 2002); 3) la permanencia con una sola hembra todo el tiempo, contrariamente a lo observado en especies monógamas, donde el exceso de hembras resulta en agregaciones poligínicas (Smith et al. 1982); y 4) efecto latitudinal en la producción de $\mathrm{T}$, al aumentar la latitud se han observado temporadas reproductivas cortas y bajas concentraciones de $\mathrm{T}$ en respuesta a la inestabilidad latitudinal de los ambientes (Moore et al. 2005, Garamszegi et al. 2008). En general, los patrones fisiológicos asociados a los sistemas de apareamiento indican que los machos tienen fluctuaciones en las concentraciones de $\mathrm{T}$ en respuesta al conflicto de asignaciones entre el esfuerzo reproductivo y el esfuerzo parental (Wingfield 1984, Wingfield et al. 1990, Peters 2002, Goymann et al. 2007). Los machos monógamos presentan una elevación de $\mathrm{T}$ al comienzo del periodo reproductivo que activa el comportamiento sexual y decrece después de la cópula que es lo que observamos en este estudio. En cambio, los machos poligínicos buscan copular con el mayor número de hembras posible durante el periodo reproductivo, lo que mantiene concentraciones elevadas y constantes de T que disminuyen sólo al final de la temporada (Wingfield et al. 1990, Nelson 2000, Goymann et al. 2007).

Perfil hormonal de hembras. Existieron cambios en las concentraciones de $\mathrm{E}_{2 \mathrm{f}}$ a lo largo del tiempo; las hembras H1 y $\mathrm{H} 2$ tuvieron incrementos en el periodo no reproductivo posiblemente como activación gonadal (Blas et al. 2010, Williams 2012, Gaur et al. 2013). Estos aumentos de $E_{2 f}$ se dieron cuatro meses antes de la puesta del primer huevo en ambas hembras, y coinciden con la actividad ovárica observada en la laparoscopía del sexado. No obstante, la H3 mostró un solo incremento, durante el resto del periodo de muestreo se mantuvieron bajos. Sugerimos que las concentraciones de $\mathrm{E}_{2 \mathrm{f}}$ eran suficientes para mantener una actividad gonadal, aún sin que mostrara comportamiento reproductivo (Wingfield et al. 1990) y fuera sexualmente adulta (Cornejo 2008). Los comportamientos agonísticos de la H4 hacia la H3 (obs. per., Cornejo 2009), pudieron haber ocasionado supresión de la actividad ovárica (Wielebnowski et al. 2002). El territorio de anidación de hembras $O$. derbianus silvestres se estima entre 0.9 y 1.8 hectáreas (González-García 1997), espacio que no tenían en absoluto los albergues. Este trabajo sugiere que los albergues de reproducción y/o exhibición inapropiados (p. e. menores en tamaño al territorio individual observado en libertad) pueden inducir comportamientos agonísticos que producen la liberación de cortisol e inhiben la actividad gonadal, dando como resultado alteraciones en el comportamiento y falla reproductiva (Kleiman 1980, Wingfield et al. 1991, Wielebnowski et al. 2002).

Relación estradiol: testosterona. La cuantificación de las dos hormonas, y su cociente $\mathrm{E}_{2 \mathrm{f}} / \mathrm{T}_{\mathrm{f}}$, podrían ser utilizados para determinar el sexo en esta especie como ocurre en otras aves monomórficas (Czekala \& Lasley 1977). La interacción de las hormonas reproductivas $\left(\mathrm{E}_{2} / \mathrm{T}\right)$ controla el sistema reproductor e influye en el éxito reproductivo del individuo (Gee 1995). Así se pudo estimar la actividad gonadal de los machos, que a pesar de que no existieron diferencias en las concentraciones de $\mathrm{T}_{\mathrm{f}}$, el M3 no mostró ninguna conducta sexual de cortejo y mantuvo un 
comportamiento "nervioso" que podría denotar estrés, ya que emitía vocalizaciones de alerta (González-García 1984, 1995). En la laparoscopía de sexado, estos machos mostraron actividad gonadal reflejada por agrandamiento y gran irrigación sanguínea de los testículos. El M3 no desarrolló comportamiento sexual, pero es posible que la concentración de $\mathrm{T}$ por debajo de los niveles reproductivos haya sido suficiente para una producción espermática viable (Wingfield et al. 1990, Peters 2002). Además, desarrolló concentraciones de $\mathrm{E}_{2 \mathrm{f}}$ más altas que los otros machos. Aunque fue capturado en vida libre en 1992 y se calculó su edad a partir de esa fecha, es probable que tenga una mayor edad, ya que en algunas gallináceas las concentraciones de $\mathrm{E}_{2}$ se incrementan con la senescencia (Weil et al. 1999). El incremento en $\mathrm{E}_{2}$ desarrolla retroalimentación negativa sobre la T, pero las concentraciones bajas de T permiten una producción espermática normal sostenida (Weil et al. 1999) y no provocan deterioro en la calidad del eyaculado (Rosenstrauch et al. 1994). Aunque este macho (M3) mostrara altas concentraciones de $E_{2 f}$ se separó del grupo de las hembras de acuerdo a la relación $E_{2 f} / T_{f}$, sugiriendo que gonadalmente es apto para reproducción. Esto ha sido confirmado en los reportes del Studbook de la especie (Cornejo 2008) donde indica que este individuo y la hembra $\mathrm{H3}$ tuvieron descendencia 3 años posteriores a este estudio (16/04/2005). La interacción con diferentes variables como el fotoperiodo, abundancia de alimento, temperatura y estímulos sociales pueden afectar o estimular fisiológicamente en la reproducción modulando la maduración o regresión gonadal (Dawson 2008). Los demás individuos mostraron relaciones $E_{2 f}$ $\mathrm{T}_{\mathrm{f}}$ correspondientes a su sexo asociadas a una actividad gonadal efectiva; se han producido 16 y 3 pollos por las parejas H1/M1 y H2/M2, respectivamente. No existen informes, sin embargo, de que la $\mathrm{H} 4$ se haya reproducido.

En conclusión, aunque son pocos los individuos estudiados, se sugiere que es posible determinar el sexo hormonalmente en O. derbianus, así como la estimación del estado y cambios en la actividad y función reproductiva. Asimismo, se sugiere que el manejo inadecuado y diseño de albergues puede resultar en la falla reproductiva. Los programas de reproducción en cautiverio deben de considerar el sistema reproductivo y la evaluación del estado reproductivo de los individuos y el diseño de albergues, para explotar su potencial fisiológico y reproductivo en beneficio de las especies en peligro, principalmente. La evaluación de la condición reproductiva puede ser una herramienta para la toma de decisiones en programas de reproducción en cautiverio, y en dado caso proponer técnicas de reproducción asistida (Staley et al. 2007). Por mucho tiempo, estas técnicas han sido exitosamente aplicadas en otras especies de aves en peligro de extinción
(Brock 1991, Durrant et al. 1995) y con la ayuda de las estimaciones de concentraciones hormonales, podría realizarse un protocolo para el pavón.

Agradecimientos. Al Consejo Nacional de Ciencia y Tecnología (CONACyT) por la beca número 159239 otorgada a LMGF. Agradecemos a Margarita Martínez (Centro de Investigaciones Fisiológicas, Universidad Autónoma de Tlaxcala, Tlaxcala, México) y a Marco A. Sánchez (Laboratorio de Neurofisiología, Universidad Autónoma de Querétaro, Querétaro, México) por su invaluable apoyo durante el trabajo de laboratorio. A Alberto González Romero por su aportación con comentarios iniciales y a Sonia Gallina por su apoyo en los análisis de conjuntos difusos. También a la dirección y el personal del zoológico Africam Safari por las facilidades logísticas y el acceso a los animales para la realización de este trabajo. Por último, a dos revisores anónimos por sus acertados comentarios al manuscrito.

\section{LITERATURA CITADA}

Álvarez del Toro, M. 1976. Datos biológicos del pavón (Oreophasis derbianus G. R. Gray). Universidad Autónoma de Chiapas, 1: 4354.

Bercovitz, A. B. 1987. Avian sex determination techniques. Pp. 533550, In: Burr, E. W. (Ed.). Companion birds medicine. Ames: Iowa State University Press.

Bermúdez-Humarán, L. G., Chávez-Zamarripa, P., Guzmán-Velasco, A., Leal-Garza, C. H. \& Montes de Oca-Luna, R. 2002. Loss of restriction site DdeI, used for avian molecular sexing, in Oreophasis derbianus. Reproduction in Domestic Animals, 37: 321-323.

Bishop, C. M. \& Hall, M. R. 1991. Non-invasive monitoring of avian reproduction by simplified faecal steroid analysis. Journal of Zoology London, 224: 649-668.

Blas, J., López, L., Tanferna, A., Sergio, F. \& Hiraldo, F. 2010. Reproductive endocrinology of wild, long-lived raptors. General and Comparative Endocrinology, 168: 22-28.

Bosserman, R. W. \& Ragade, R. K. 1982. Ecosystems analysis using fuzzy set theory. Ecological Modelling, 16: 191-208.

Brock, M. K. 1991. Semen collection and artificial insemination in the Hispaniolan parrot (Amazona ventralis). Journal of Zoo and Wildlife Medicine, 22: 107-114.

Brooks, D. M. \& Strahl, S. D. 2000. Curassows, guans and chachalacas. Status survey and conservation. Action plan for cracids 2000-2004. IUCN/SSC Cracids Specialist Group. IUCN, Gland, Switzerland and Cambridge, UK. viii + 182 pp.

Casanueva, F. F. \& Vázquez, J. A. 1995. Endocrinología clínica. Ediciones Díaz de Santos, S.A. Madrid, España.

Cockrem, J. F. \& Rounce, J. R. 1994. Faecal measurements of oestradiol and testosterone allow the non-invasive estimation of plasma steroid concentrations in the domestic fowl. British Poultry Science, 35: 433-443.

Cockrem, J. F. \& Rounce, J. R. 1995. Non-invasive assessment of the annual gonadal cycle in free-living Kakapo (Strigops habroptilus) using fecal steroid measurements. The Auk, 112: 253-257.

Cornejo, J. 2008. Studbook Internacional del pavón (Oreophasis derbianus). $4^{\text {a }}$ Edición. Africam Safari / Schubot Exotic Bird Health Center. Alcance 31 de Diciembre del 2008.

Cornejo, J. 2009. Breeding programme for Horned Guan, Oreophasis derbianus, at Africam Safari, Mexico. International Zoo Yearbook, 43: 136-148. 
Czekala N. M. \& Lasley, B. L. 1977. A technical note on sex determination in monomorphic birds using faecal steroids analysis. International Zoo Yearbook, 17: 209-211.

Dawson, A. 2008. Control of the annual cycle in birds: endocrine constraints and plasticity in response to ecological variability. Philosophical Transactions of the Royal Society B, 363: 1621-1633.

Delacour, J. \& Amadon, D. 1973. Curassows and related birds. The American Museum of Natural History. Chanticleer Press, Inc. NY, USA.

Donham, R. S. 1980. Annual cycle of plasma luteinizing hormone and sex hormones in males and females Mallards (Anas platyrhynchos). Biology of Reproduction, 21: 1273-1285.

Durrant, B., Burch, C. D., Yamada, J. K. \& Good, J. 1995. Seminal characteristics and artificial insemination of Chinese pheasants, Trogopan temmincki, Lophophorus impeyanus and Lophophorus lhuysii. Zoo Biology, 14: 523-531.

Emlen, S. T. \& Oring, L. W. 1977. Ecology, sexual selection, and the evolution of mating systems. Science, 197: 215-222.

Equihua-Zamora, M. 2001. Fuzzy clustering tool, versión 2 Beta. Instituto de Ecología, A.C.

Estudillo, L. J. 1997. Los crácidos: la familia de aves Neotropicales mas amenazada de extinción y su posible restablecimiento por la reproducción en cautiverio, pp: 117-123. In: Strahl, S. D., D. M. Brooks, A. J. Begazo, G. Sedaghafkish \& F. Olmos (eds.). The Cracidae: their biology and conservation. Hancock House Publishers.

Garamszegi, L. Z., Hirschenhauser, K., Bókony, V., Eens, M., Hurtrez-Boussès, S., Møller, A.P., Oliveira, R. F. \& Wingfield, J. C. 2008. Latitudinal distribution, migration, and testosterone levels in birds. The American Naturalist, 172: 533-546.

Gaur, U., Tripathi, B. K. \& Shrivastava, S. K. 2013. Study of photoperiodism on gonadal weight in a Readhead bunting under natural day length [Ndl]. Asian Journal of Experimental Biological Science, 4: 74-78.

Gee, G. F. 1995. Avian reproductive physiology, pp. 241-262. In: Gibbons Jr. E., B. Durrant \& J. Demarest (Eds.). Conservation of endangered species in captivity. State University of New York Press, Albany.

González-García, F. 1984. Aspectos biológicos del pavón Oreophasis derbianus G. R. Gray (Aves: CRACIDAE) en la Reserva Natural “El Triunfo”, Municipio de Ángel Albino Corzo, Chiapas, México. Tesis profesional. Universidad Veracruzana. Xalapa, Veracruz, México. 83 pp.

González-García, F. 1995. Reproductive biology and vocalizations of the horned guan Oreophasis derbianus in Mexico. The Condor, 97: 415-426.

González-García, F. 1997. Conducta de anidación del pavón (Oreophasis derbianus: Aves, Cracidae) en la Reserva de la Biosfera el Triunfo, Chiapas, México, pp. 418-422. In: Strahl, S. D., S. Beaujon, D. M. Brooks, A. J. Begazo, G. Sedaghatkish \& F. Olmos (Eds.). The Cracidae: Their biology and conservation. Hancock House Publishers.

González-García, F. 2001. Estado de Conservación del pavón Oreophasis derbianus en el campo y en cautiverio en México, pp. 147-166. In: Brooks, D. M. \& F. González-García (Eds.). Biology and Conservation of Cracids in the New Millenium. Misc. Publ. HMNS 2, Houston, TX.

Goymann, W., Landys, M. M. \& Wingfield, J. C. 2007. Distinguishing seasonal androgen responses from male-male androgen responsiveness-revisiting the challenge hypothesis. Hormones and Behavior, 51: 463-476.
Griffiths, R., Double, M. C., Orr, K. \& Dawson, R. J. G. 1998. A DNA test to sex most birds. Molecular Ecology, 7: 1071-1075.

Johnson, A. L. 1999. Reproduction in the female, pp. 569-596. In: G. Causey Whittow (ed), Sturkie's Avian physiology, fifth edition. Academic Press.

Kirby, J. D. \& Froman, D. P. 1999. Reproduction in the male birds, pp. 597-615. In: G. Causey Whittow (Ed.), 1999. Sturkie's Avian physiology, fifth edition. Academic Press.

Kleiman, D. 1980. The sociobiology of captive propagation, pp. 243261. In: Soulé, M. E. \& B. A. Wilcox (Eds.). Conservation biology. Sunderland, MA. Sinauer Associates.

Kricka, L. J. 1984. Luminescent immunoassay for hormones. Journal of Endocrinology, 102: suppl, Abstract 68.

Kricka, L. J. 1991. Sensitive detection systems, chemiluminescent and bioluminescent techniques. Clinical Chemical, 37:1472-1481.

Hasse, E. \& Donham, R. S. 1980. Hormones and domestication, pp. 549-565. In: Epple, A. \& M.H. Stetson (Eds.). Avian endocrinology. New York, Academic Press.

Lasley, B. L. \& Kirkpatrick, J. F. 1991. Monitoring ovarian function in captive and free-ranging wildlife by means of urinary and fecal steroids. Journal of Zoo and Wildlife Medicine, 22: 23-31.

Leisler, B., Winkler, H. \& Wink, M. 2002. Evolution of breeding systems in Acrocephaline warblers. The Auk, 119: 379-390.

Moore, I. T., Bonier, F. \& Wingfield, J. C. 2005. Reproductive asynchrony and population divergence between two tropical bird populations. Behavioral Ecology, 16: 755-762.

Nelson, R. J. 2000. An introduction to behavioral endocrinology. 2a. Edition. Sinauer Associates, Inc. Publishers. Sunderland, Massachusetts, $724 \mathrm{pp}$.

Peters, A. 2002. Testosterone and the trade-off between mating and parental effort in extra pair-mating superb fairy-wrens. Animal Behaviour, 64: 103-112.

Ptak, S. E. \& Lachmann, M. 2003. On the evolution of polygyny: a theoretical examination of the polygyny threshold model. Behavioral Ecology, 14: 201-211.

Ritchie, B. W., Harrison, G. J. \& Harrison, L. R. 1994. Avian medicine: principles and application. Wingers Publishing, Inc. Lake Worth, Florida, USA.

Rosenstrauch, A., Degen, A. A. \& Friedländer, M. 1994. Spermatozoa retention by Sertoli cells during the decline in fertility in aging roosters. Biology of Reproduction, 50: 129-136.

Sheppard, C. 1995. Captive propagation and avian conservation, pp. 227-240. In: Gibbons Jr. E., B. Durrant, \& J. Demarest (Eds.). Conservation of endangered species in captivity. State University of New York Press, Albany.

Schwarzenberger, F. \& Brown, J. L. 2013. Hormone monitoring: an important tool for the breeding management of wildlife species. Wiener Tierärztliche Monatsschrift-Veterinary Medicine Austria, 100: 209-225.

Smith, J. N., Yom-Tov, Y. \& Moses, R. 1982. Polygyny, male parental care, and sex ratio in song sparrows: an experimental study. The Auk, 99: 555-564.

Staley, A. M., Blanco, J. M., Dufty Jr., A. M., Wildt, D. E. \& Monfort, S. L. 2007. Fecal steroid monitoring for assessing gonadal and adrenal activity in the golden eagle and peregrine falcon. Journal of Comparative Physiology B, 177: 609-622.

StatSoft, Inc. 2004. STATISTICA (data analysis software system), version 7. www.statsoft.com.

Vaurie, C. 1968. Taxonomy of Cracidae (Aves). Bulletin of American Museum of the Natural History 138:133-259. 
von Ende, C. N. 2001. Repeated-measure analysis: Growth and other time dependent measures, pp. 134-157. In: Scheiner, S. \& I. Gurevitch (eds). The design and analysis of ecological experiments. Oxford University Press.

Weeks, I. \& Woodhead, J. 1984. Chemiluminescene immunoassay. Journal of Clinic Immunology, 7: 82-89.

Weil, S., Rozenboim, I., Degen, A. A., Dawson, A., Friedländer, M. \& Rosenstrauch, A. 1999. Fertility decline in aging roosters is related to increase testicular and plasma levels of estradiol. General and Comparative Endocrinology, 115: 23-28.

Wielebnowski, N. C., Ziegler, K., Wildt, D. E., Lukas, J. \& Brown, J. L. 2002. Impact of social management on reproductive, adrenal and behavioural activity in the cheetah (Acinonyx jubatus). Animal Conservation, 5: 291-301.
Williams, T. D. 2012. Physiological adaptation for breeding in birds. Princeton University Press, New Jersey.

Wingfield, J. C. 1984. Androgens and mating system: testosteroneinduced polygyny in normal monogamous birds. The Auk, 101: 665-671.

Wingfield, J. C., Hegner, R. E., Dufty Jr., A. M. \& Ball, G. F. 1990. The "challenge hypothesis": theoretical implications for patterns of testosterone secretion, mating system, and breeding strategies. American Naturalist, 136: 829-846.

Wingfield, J. C., Hegner, R. E. \& Lewis, D. M. 1991. Circulating levels of luteinizing hormone and steroid hormones in relation to social status in the cooperatively breeding white-crowned sparrow weaver, Plocepasser mahali. Journal of Zoology London, 225: 43-58. 